\title{
Parainfectious encephalitis in COVID-19: "The Claustrum Sign”
}

\author{
Frédéric Zuhorn ${ }^{1}$ (1) Hassan Omaimen ${ }^{2} \cdot$ Bertram Ruprecht $^{3} \cdot$ Christoph Stellbrink $^{4} \cdot$ Michael Rauch $^{1}$. \\ Andreas Rogalewski ${ }^{1} \cdot$ Randolf Klingebiel $^{2}$ • Wolf-Rüdiger Schäbitz ${ }^{1}$
}

Received: 6 July 2020 / Revised: 21 August 2020 / Accepted: 24 August 2020 / Published online: 3 September 2020

(c) Springer-Verlag GmbH Germany, part of Springer Nature 2020

Dear Sirs,

Recent evidence supports a neurotropic activity of SARS$\mathrm{CoV}-2$, causing encephalopathy with various neurological symptoms. Apart from acute necrotizing encephalopathy (ANE), imaging findings on brain MRI scans in COVID19 encephalopathy were reported as being unspecific and equivocal. Herein, we present a severe case of SARSCoV-2 disease, with evidence of parainfectious autoimmune encephalitis, causing a specific and previously undescribed MRI pattern.

A 54-year-old male, Caucasian outpatient was diagnosed with COVID-19 by a positive RT-PCR test for SARS-CoV-2 using a nasopharyngeal swab. Initial symptoms included weakness, fever and unproductive cough. The patient was admitted to hospital on day 3 following clinical deterioration and presented with neuropsychiatric symptoms (aggressiveness, disorientation, stupor) even before respiratory deterioration, suggestive for encephalitic syndrome. There were no signs of epileptic activity or seizure-like symptoms. Medical history revealed arterial hypertension, obesity (BMI $34 \mathrm{~kg} /$ $\mathrm{m}^{2}$ ) and obstructive sleep apnea syndrome. Lung sounds and blood gas analysis were unremarkable ruling out hypoxemia. Chest CT disclosed multilobular, bilateral lung infiltrates. Laboratory tests results are presented in (Table 1) and

Randolf Klingebiel and Wolf-Rüdiger Schäbitz both authors contributed equally to this work.

Frédéric Zuhorn

frederic.zuhorn@evkb.de

1 Department of Neurology, Evangelisches Klinikum Bethel EvKB, Bielefeld, Germany

2 Department of Neuroradiology, Evangelisches Klinikum Bethel EvKB, Bielefeld, Germany

3 Department of Pulmonary Medicine, Klinikum Bielefeld, Bielefeld, Germany

4 Department of Cardiology and Intensive Care Medicine, Klinikum Bielefeld, Bielefeld, Germany included positive serum antibody indices for SARS-CoV-2. On day 4, respiratory deterioration required endotracheal intubation and treatment of bacterial superinfection was started according to antibiogram. Eight days later, the patient could be extubated and PCR tests were negative for SARSCoV-2. Yet, the patient continued to show concentration difficulties and delirious behavior. Subsequent MRI (Fig. 1ac) revealed signal alterations within the claustrum/external capsule region, showing reduced diffusion. Cerebrospinal fluid (CSF) analysis disclosed a mild lymphocytic pleocytosis with negative test results for common neurotropic viruses. Tests in serum and CSF were also negative for various antineuronal antibodies. The patient recovered and was discharged with only mild cognitive impairment.

Follow-up has been carried out four months later showing a normalization in cell count of CSF and improvement of MRI findings, although the claustrum lesions persisted. Clinically, his neurological and cognitive status was normal.

Our case is characterized by evidence of parainfectious autoimmune encephalitis in the context of severe COVID-19 pneumonia. Clinically, the patient presented with various neuropsychiatric symptoms, which were reported before in other COVID-19 patients with encephalopathy [1]. Neither SARS-CoV-2 itself nor antibodies against the virus were found positive in the CSF, precluding direct viral CNS infection. Comprehensive laboratory tests ruled out antineuronal antibodies as well as common infectious causes of encephalitis, altogether supporting the diagnosis of parainfectious autoimmune encephalitis. In addition, the diagnostic criteria for possible autoimmune encephalitis as proposed by Gaus et al. were met [2].

While immunological markers remained unspecific and imaging findings of acute necrotizing encephalitis were absent in our patient, brain MRI disclosed a unique pattern, a.k.a. the claustrum sign. Previously, this sign has been coined in MRI studies of autoimmune epilepsy, where an immune-inflammatory-mediated encephalopathy is suspected [3]. The claustrum is known to play a crucial role in regulating consciousness [6] correlating well to the 
Table 1 Laboratory findings in blood and CSF (in the inpatient course)

\begin{tabular}{|c|c|c|}
\hline Measure (blood) & Reference range & Result \\
\hline White blood cell count (G/L) & $4.40-11.30$ & 6.7 \\
\hline Red blood cell count (T/L) & $4.50-5.90$ & 4.72 \\
\hline Lymphocytes (\%) & $10-50$ & 13 \\
\hline $\mathrm{LDH}(\mathrm{U} / \mathrm{L})$ & $<248$ & 328 \\
\hline D-dimers $(\mu \mathrm{g} / \mathrm{l})$ & $<500$ & 2001 \\
\hline Myoglobin $(\mu \mathrm{g} / \mathrm{l})$ & $23.0-72.0$ & 370.9 \\
\hline IL-6 (pg/ml) & $<8.0$ & 90.2 \\
\hline $\mathrm{CRP}(\mathrm{mg} / \mathrm{l})$ & $<5.0$ & 93.3 \\
\hline SARS-CoV-2-IgA antibody (indices) & $<0.8-1.0$ & 10.9 \\
\hline SARS-CoV-2-IgG antibody (indices) & $<0.8-1.0$ & 13.2 \\
\hline Measure (CSF) & Reference range & Result \\
\hline CSF aspect & Clear & Clear \\
\hline White blood cell count $(/ \mu \mathrm{l})$ & $<5$ & 9 \\
\hline Red blood cell count $(/ \mu \mathrm{l})$ & $<100$ & $<100$ \\
\hline Monocytes (\%) & & 12 \\
\hline Lymphocytes (\%) & & 88 \\
\hline Total Proteins (mg/l) & $180-430$ & 396 \\
\hline Glucose (mg/dl) & $<75$ & 57 \\
\hline Lactate $(\mathrm{mmol} / \mathrm{l})$ & $1.70-2.60$ & 1.65 \\
\hline Isoelectrofocusing & Normal & Normal \\
\hline Advanced virus measures (CSF) & Reference range & Result \\
\hline DNA-PCR of Herpes Simplex Virus, Varicella-Zoster Virus, Cytomegaly and Epstein Barr Virus & Negative & Negative \\
\hline RT-PCR for SARS-CoV-2 Virus & Negative & Negative \\
\hline SARS-CoV-2-IgA antibody & Negative & Negative \\
\hline SARS-CoV-2-IgG antibody & Negative & Negative \\
\hline Measles IgG & $<1.4$ & 1.1 \\
\hline Rubella IgG & $<1.4$ & 1.3 \\
\hline Herpes Simplex Virus 1/2 IgG & $<1.4$ & 1.1 \\
\hline Varicella Zoster Virus IgG & $<1.4$ & 1.1 \\
\hline \multicolumn{3}{|l|}{ Advanced antineuronal antibody measures (serum and CSF) } \\
\hline $\begin{array}{l}\text { GAD65, NMDA-, and GABA-B-receptor, IgLON5, AMPA-R subtype 2, DPPX, LGI1, CASPR2, Glycine-receptor, } \\
\text { mGluR5, mGluR1, Amphiphysin, CV2/CRMP5, Ma2/Ta, Hu, Ri, Yo, Zic4, Recoverin, Sox1, Titin, DNER/Tr }\end{array}$ & Negative & Negative \\
\hline
\end{tabular}

clinical findings of impaired levels of consciousness in the presented case.

In autoimmune epilepsy, the claustrum signals normalized in the majority but not in all patients [4], suggesting a varying severity of claustrum damage. This is confirmed by reduced diffusion in the first MRI scan of our patient, heralding irreversible tissue damage (as proven by the 4-month MRI follow-up). At no point in time, there was evidence for other causes of diffusion reduction, i.e., hypoxemia or status epilepticus. Comparable claustrum lesions have also been reported in the context of autoimmune encephalitis without epileptic or anoxic episodes, supporting inflammation as a decisive factor [5].
A particular vulnerability of claustral neurons to hypoxic stress has been shown [7], without relating to the inflammatory pathogenesis of our MRI findings. Yet, astrocyte proliferations and microglia/macrophage infiltrations of the claustrum have been observed in non-herpetic encephalitis [8]. To which extent other pathomechanisms, such as encephalitic hypermetabolism as known from the striatum [9]. Additionally, compromise the claustrum remains speculative.

Common MRI findings in a recent study of COVID19 encephalopathy were cortical signal abnormalities on FLAIR images (37\%), accompanied by diffusion reduction, leptomeningeal enhancement and cortical blooming artifacts in some cases. These imaging findings, termed by the authors themselves as "rather unspecific", did not allow 

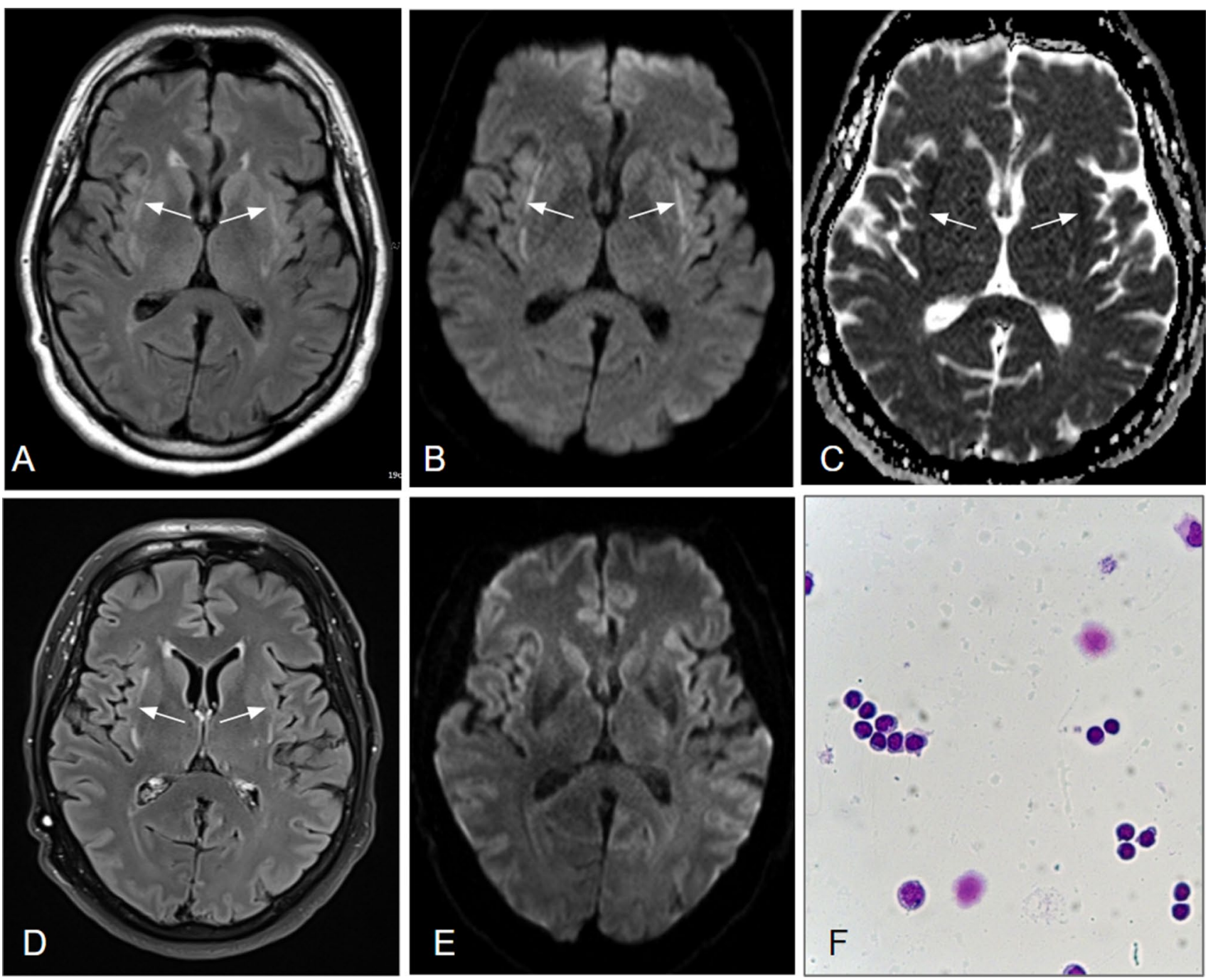

Fig. 1 a-f MR scans 2 weeks (a-c) and 4 months (d-e) post symptom onset as well as CSF cytology (f): On axial FLAIR (a), Diffusion-weighted images (b) and the corresponding ADC map (c), bilateral signal alterations within the external capsule/claustrum regions are depicted (arrows), indicative of reduced diffusion. On follow-up

imaging (d-e), the FLAIR-hyperintensities persist (d) whereas tissue diffusion has normalized (e). CSF-cytology (f) showed a slightly elevated cell count $(9 / \mu \mathrm{l})$ with a lymphocytic predominance $(88 \%$ lymphocytes, $12 \%$ monocytes). A meaningful plasmacytic transformation was not observed, the monocytes being only slightly activated

differentiation among various possible causes such as viral neurotropism, cytokine storm syndrome, hypoxia, subclinical seizures or critical illness-related encephalopathy [10].

MRI findings in COVID-19 encephalitis, especially when suggesting autoimmune encephalopathy may imply therapeutic interventions, such as immunosuppressive therapy. Recently, progressive clinical improvement along with a reduction of inflammatory CSF parameters has been observed in COVID-19 encephalitis, following high-dose steroid treatment [11].

In summary, a previously undescribed imaging pattern in parainfectious COVID-19 encephalitis is presented that bears a strong resemblance to MRI findings in autoimmune encephalitic syndromes, such as known from epileptic or

encephalitis caused by antineuronal antibodies. This claustrum sign should be added to the still limited knowledge of encephalitic imaging patterns in COVID-19, as it most probably represents an autoimmune phenomenon that might progress from reversible signal changes to permanent tissue damage and thus may trigger appropriate as well as timely therapy.

Author contributions FZ is lead author, analyzed and interpreted the collected data and literature, designed and wrote the manuscript. HO\&AR participated in the design and coordination of the manuscript. $\mathrm{BR} \& \mathrm{CS}$ helped in drafting the manuscript. MR\&RK provided figures and data and revised the manuscript for important intellectual content. 
RK\&WRS are senior authors, revised the manuscript critically and gave final approval of the version of the manuscript.

Funding The authors received no financial support for the research, authorship and publication of this article.

\section{Compliance with ethical standards}

Conflicts of interests The authors declare that they have no conflict of interest.

Ethical standard For this type of study, formal consent is not required.

Informed consent Written informed consent was obtained from the patient for publication of this case report and any accompanying images. A copy of the written consent is available for review by the Editor of this journal.

Availability of data and material We take full responsibility for the data, the analyses, and interpretation, and that we have full access to all the data.

\section{References}

1. Helms J, Kremer S, Merdji H et al (2020) Neurologic features in severe SARS-CoV-2 infection. N Engl J Med. https://doi. org/10.1056/NEJMc2008597(PublishedonlineApril15)

2. Grau $S$ et al (2016) A clinical approach to diagnosis of autoimmune encephalitis. Lancet Neurol 15(4):391-404. https://doi. org/10.1016/S1474-4422(15)00401-9
3. Specchio N, Pietrafusa N (2015) New-onset refractory status epilepticus and febrile infection-related epilepsy syndrome. Dev Med Child Neurol. https://doi.org/10.1111/dmcn.14553

4. Crick FC, Koch C (2005) What is the function of the claustrum? Philos Trans R Soc Lond B Biol Sci 360(1458):1271-1279. https ://doi.org/10.1098/rstb.2005.1661

5. Steriade $\mathrm{C}$ et al (2017) Epilepsia Open 2(4):476-480

6. Hiraga A et al (2014) Voltage-gated potassium channel antibody-associated encephalitis with claustrum lesions. Intern Med 53(19):2263-2264

7. Alturkustani M, Ang LC (2018) Claustral neurons are vulnerable to ischemic insults in cardiac arrest encephalopathy. Int J Clin Exp Pathol 11(5):2735-2741

8. Okamoto K, Yamazaki T, Banno H, Sobue G, Yoshida M, Takatama M (2008) Neuropathological studies of patients with possible non-herpetic acute limbic encephalitis and so-called acute juvenile female non-herpetic encephalitis. Intern Med 47(4):231-236

9. Rey C, Koric L, Guedj E et al (2012) Striatal hypermetabolism in limbic encephalitis. J Neurol 259(6):1106-1110. https://doi. org/10.1007/s00415-011-6308-2

10. Kandemirli SG, Dogan L, Sarikaya ZT et al (2020) Brain MRI findings in patients in the intensive care unit with COVID-19 infection. Radiology 5:201697. https://doi.org/10.1148/radio 1.2020201697(Published online May 8)

11. Pilotto A, Odolini S, Stefano Masciocchi S et al (2020) Steroidresponsive encephalitis in Covid-19 disease. Ann Neurol. https:// doi.org/10.1002/ana.25783(Published online May17) 